\title{
Chapter 21 \\ Determinants of Loyalty Intention in Portuguese Mobile Market
}

\author{
Maria Borges Tiago \\ University of the Azores, Portugal \\ Francisco Amaral \\ University of the Azores, Portugal
}

\begin{abstract}
Our work conceptualizes and highlights the determinants of customers'loyalty in the Portuguese mobile market. We raise questions about the interrelationships of the cost and values dimensions and the consequences of these relationships on customer satisfaction and trust and consequently loyalty among different operators, addressing some recent models. By organizing and synthesizing the major research streams and tests empirically a conceptual framework through a SEM, with data gather in a survey of Portuguese clients, the present study advances knowledge on the nature of the relative importance of different components of loyalty to mobile communications operators. Some useful preliminary insights were produced related to customers'retention process in primary mobile operator, which appears strongly related to price/quality, followed by the emotional connection to the operator staff and others clients. Nonetheless, a considerable number of issues were left for future research, including the possibility of extending the investigation to other countries.
\end{abstract}

\section{INTRODUCTION}

With the emergence of high-speed wireless network technologies and the escalating market penetration of mobile phones, the need to analyze customer behavior is growing. The mobile communication phenomenon is unique in the histories of both the telecommunication and the consumer

DOI: 10.4018/978-1-60960-042-6.ch021 electronics markets. In less than a decade, millions of people have begun to use mobile phones.

Ever since the mid-1990s, mobile phones have becomeubiquitous in developed economies. While in 1997 only 215 million people worldwide were using mobile communication devices, by 2001 this number had grown to 961 million and to 2.7 billion by 2006 (Union, 2009).

Mobile communication was the first telecommunication segment to be liberalized in Portugal, 
following the breakthrough of GSM technology. Due to strong competition, the market has been growing, reaching its present penetration rate of over $100 \%$. Portugal has been a pioneer in the massive adoption of mobile communications; it was the first country to adopt pre-paid cards and more recently to adopt mobile Internet access programs for all students in elementary and secondary school ("e-escolinhas" and "e-escolas", respectively).

The Autoridade Nacional de Comunicações (ANACOM, 2007), which supervises Portugal's communications sector, has published a quality report of voice, video-telephony services and network coverage of GSM and WCDMA, concluding that there are no significant differences in the quality of services rendered by the various operators. For example, the Portuguese government negotiated identical prices and technical characteristics from all Portuguese mobile operators for the "e-escolinhas" and "e-escolas" programs.

In an intensely mutating market where the introduction of $3 \mathrm{G}$ technologies, technological convergence, and the emergence of news mobile operators permits constant offerings of innovative services, customers' loyalty is very important for a mobile operator's success. Thus, the determinants of customer loyalty are essential for the commercial success, not only for traditional mobile operators, but also for the virtual ones. From a research standpoint, it raises an important question: what are the determinants of mobile customers' loyalty?

The objective of the present work is to elaborate on this understanding by distinguishing the different types of cost, values and quality dimensions in the literature. It establishes conceptual frameworks which consider all these components and their impact on loyalty in the mobile market. Drawing on a detailed reading of Lim, Widdows, and Park (2006) and Junglas and Watson (2008), such a conceptual framework was established and several hypotheses related to the customer loyalty determinants were formulated: price and quality, social value, economic value, emotional value, processing costs, emotional costs and financial costs.

Ouranalysis does notattempt to "prove" a set of claims about consumer behavior, but to develop a new interpretation, one which offers fresh insights and understanding. With this purpose in mind, data was collected and the sample obtained consists of 262 customers of Portuguese mobile operators. Using a structural equation analysis, we explore the relationship among price/quality, social value, economic value, emotional value, processing costs, emotional costs and financial costs, trying to identify the main drivers of customers' loyalty.

In general, our findings support the conceptual framework. The results sustain our conceptualization for the loyalty construct and allow us to conclude that Portuguese customers will remain with the same mobile operator primarily because of price/quality, followed by the emotional connection to the operator staff and to its other clients. However, the direct determinants of customer loyalty are satisfaction and trust.

Despite these findings, the operators, regardless of their typology (non-virtual or virtual), should concern themselves primarily with establishing a basis of loyalty with current clients and secondarily with acquiring new customers, even in mature markets. Moreover, the perception of loyalty determinants change with time, so operators must constantly monitor these determinants and create new ways to increase loyalty.

This research and its findings will be useful for firms intending to emulate the application of virtual mobile communications or even improve mobile communications strategies among current operators.

This chapter is organized in the following manner. The next section summarizes the literature related to customer behaviors regarding mobile operators. The third section of the paper formalizes the major points presented in the conceptual model and translates them into hypotheses. In the 
14 more pages are available in the full version of this document, which may be purchased using the "Add to Cart" button on the publisher's webpage: www.igi-global.com/chapter/determinants-loyalty-intention-portuguesemobile/50596

\section{Related Content}

\section{Electronic Journalism in Peru}

Antonio Diaz and Martin Santana (2003). Information Management: Support Systems \& Multimedia Technology (pp. 277-297).

www.irma-international.org/chapter/electronic-journalism-peru/22963/

\section{Adaptive Hypermedia for Personalised TV}

Judith Masthoff and Lyn Pemberton (2005). Adaptable and Adaptive Hypermedia Systems (pp. 246-263). www.irma-international.org/chapter/adaptive-hypermedia-personalised/4188/

An Image Clustering and Feedback-based Retrieval Framework Chengcui Zhang, Liping Zhou, Wen Wan, Jeffrey Birch and Wei-Bang Chen (2010). International Journal of Multimedia Data Engineering and Management (pp. 55-74). www.irma-international.org/article/image-clustering-feedback-based-retrieval/40985/

Building Multi-Modal Relational Graphs for Multimedia Retrieval Jyh-Ren Shieh, Ching-Yung Lin, Shun-Xuan Wang and Ja-Ling Wu (2011). International Journal of Multimedia Data Engineering and Management (pp. 19-41). www.irma-international.org/article/building-multi-modal-relational-graphs/54460/

Fast Caption Alignment for Automatic Indexing of Audio

Allan Knight and Kevin Almeroth (2010). International Journal of Multimedia Data Engineering and Management (pp. 1-17).

www.irma-international.org/article/fast-caption-alignment-automatic-indexing/43745/ 